\title{
The aggregation of Calcium Silicate Hydrate
}

\section{Nanoplatelets}

\author{
Maxime Delhorme ${ }^{\dagger}$ Christophe Labbez, ${ }^{*} \dagger$ Martin Turesson, ${ }^{\dagger}$ André Nonat, $^{\dagger}$ \\ Cliff Woodward, ${ }^{\ddagger}$ and Bo Jönsson $₫$ \\ $\dagger I C B$, UMR 6303 CNRS, Univ. Bourgogne France-Comté, F-21078 Dijon Cedex, France \\ $\ddagger$ PEMS, University of New South Wales, Canberra, 260x ACT, AUSTRALIA \\ ФiTheoretical Chemistry, Chemical Center, P.O. Box 124, S-221 00, Lund, Sweden \\ E-mail: christophe.labbez@u-bourgogne.fr
}

\section{Platelet and surface model}

The platelets were modeled as discs composed of spherical sites arranged on a regular hexagonal lattice. Platelet sites had a diameter of $5 \AA$. Adjacent platelet sites, $i$ and $j$, were connected with a harmonic potential with $b_{i j}=0.5 \mathrm{~nm}$ and $k_{i j}^{b}=1000 \mathrm{kJmol}^{-1} \mathrm{~nm}^{-2}$. In addition, the platelet sites were maintained in-plane by a harmonic potential on the angle $\theta_{i j k}$, between triplets of bonded sites,

$$
V_{a}\left(\theta_{i j k}\right)=\frac{1}{2} k_{i j k}^{\theta}\left(\theta_{i j k}-\theta_{i j k}^{0}\right)^{2}
$$

with $\theta_{i j k}^{0}=\pi$ and $k_{i j k}^{\theta}=10^{4} \mathrm{~kJ} / \mathrm{mol} / \mathrm{rad}^{2}$.

The infinite surface was modeled of spherical sites arranged in a square lattice with diameter equal to that of the platelets. The position of these sites were kept fixed during the simulations. The diameter of the simple ions was set to $4 \AA$. 


\section{Interaction potentials}

The interaction potential between ions and platelet sites and between ions and sites them-

selves consists of a Coulombic interaction augmented with a shifted Lennard-Jones potential,

$$
U^{L J}\left(r_{i j}\right)=4 \epsilon_{L J}\left(\left(\frac{\sigma_{L J}}{r_{i j}}\right)^{12}-\left(\frac{\sigma_{L J}}{r_{i j}}\right)^{6}\right)+\epsilon_{L J}
$$

with $\epsilon_{L J}=1 k T$ and

$$
\sigma_{L J}=\frac{\left(R_{i}+R_{j}\right)}{2^{1 / 6}}
$$

The ions and plate sites interact with the surface sites via the Coulomb potential and in addition they interact with an integrated Lennard Jones potential of the form,

$$
U_{W}^{L J}\left(h_{i}\right)=2 \pi \epsilon_{w}\left[\frac{2}{45}\left(\frac{\sigma_{w}}{h_{i}}\right)^{9}-\frac{1}{3}\left(\frac{\sigma_{w}}{h_{i}}\right)^{3}\right]
$$

where $h_{i}$ is the distance of the species $i$ to the surface, $\epsilon_{w}=0.01 k T$ and $\sigma_{w}=2.4 \AA$. The ions and platelet sites interact with the neutral surface with the same potential.

\section{MD simulation details}

All molecular dynamics (MD) simulations were performed with the simulation software GROMACS, version $4.5 .4,{ }^{1}$ using a velocity Verlet algorithm to integrate Newton's equations of motion for the freely moving species. The temperature for each species was thermostated using velocity rescaling with a stochastic term, ensuring the generation of a proper canon-

ical ensemble. ${ }^{2}$ A time step of 10 fs was employed. To account for the long range part of the electrostatic interaction, Particle-Mesh Ewald electrostatics (PME) was used with a 60 $\AA$ real space Coulomb cut-off and a Fourier spacing equal to $6 \AA$. The reciprocal sum was calculated in three dimensions, but the force and potential correction were calculated in the z-direction (normal to the charged wall) to produce pseudo two dimensional periodic condition in directions parallel to the charged wall ( $\mathrm{x}$ - and $\mathrm{y}$-directions). The $\mathrm{z}$ dimension of the 
simulation box was chosen three times larger than the slab thickness to reduce artifacts of the method. ${ }^{1}$

\section{Many platelet simulation}

To study the effect of surface charge density on the aggregation of platelets on surfaces, we simulated a dispersion of 50 freely rotating charged platelets in contact with a charged wall, systems s1-s3. The platelet and wall charges were compensated with $\mathrm{Ca}^{2+}$ counterions and extra salt ions were added to obtain a concentration of about $20 \mathrm{mM}$ for $\mathrm{Ca}(\mathrm{X})_{2}$. A starting configuration with random platelet and mobile ion coordinates was generated. The ions were then allowed to equilibrate, keeping the platelets fixed. Once the system energy was converged with respect to the static platelet configuration, the whole system, including translation and rotation of the platelets, was allowed to move. An equilibration time of 5 ns was followed by a production run lasting for 50 ns. The simulations were run in parallel on 12 processors and took about 15 days to complete in real time.

\section{Interaction of two platelets in mono- and divalent salt solutions}

Figure 1 illustrates the striking change of behavior between two rotating platelets when the salt is varied from monovalent to divalent. As known for a long time, a double layer repulsion is observed between the particles when a 1:1 salt is present in the box. Note that, this repulsion increases when increasing the size of the particles, due to the increase of the excluded volume repulsion. When introducing a 2:1 salt in the simulation box, ion correlations come into play. It results a net attraction between the particles, as it can be seen on both the force curves and the corresponding free energy curves figure 1. The later also display size dependence interactions. 

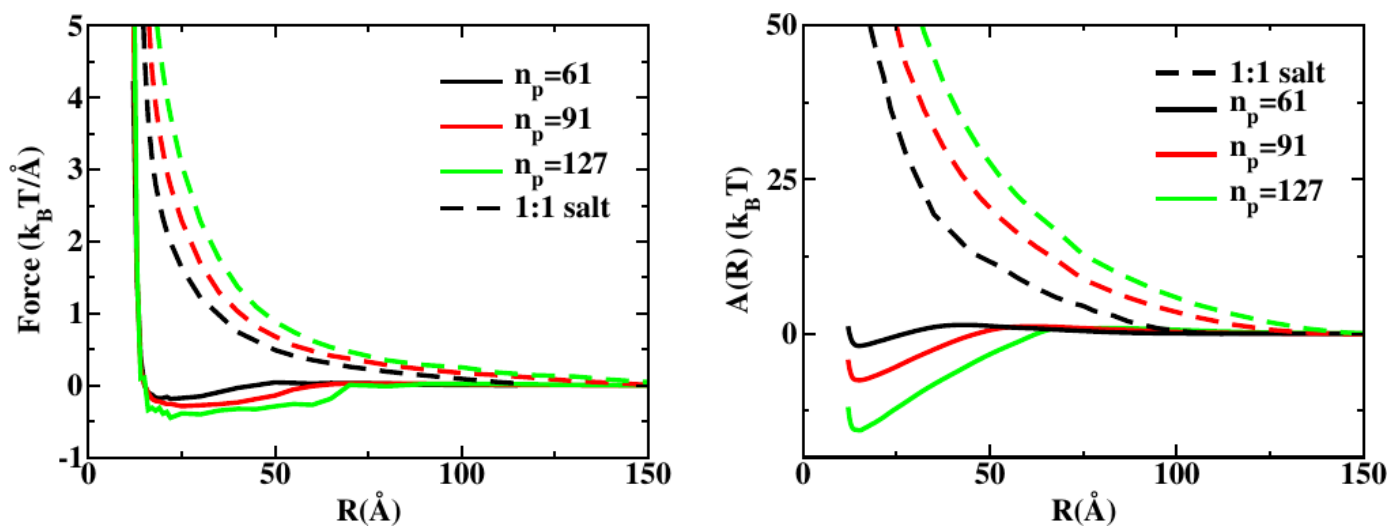

Figure 1: a) The average force between two rotating platelets with $\sigma=-1.2 \mathrm{e} / \mathrm{nm}^{2}$ in $10 \mathrm{mM}$ of a $2: 1$ salt (full lines) and a 1:1 salt (Dashed lines) at a volume fraction of 0.023. The number of sites $\left(\mathrm{n}_{p}\right)$ from 61 to 127 correspond to $9 \mathrm{~nm}$ and $13 \mathrm{~nm}$ in diameter; $\epsilon_{L J}=0.1 k_{B} T$. b) The corresponding free energy curves.

\section{References}

(1) van der Spoel, D.; Lindahl, E.; Hess, B.; van Buuren, E.; Apol, E.; Meulenhoff, P. J.;

Tieleman, D. P.; Sijbers, A. L. T. M.; Feenstra, K. A.; van Drunen, R.; Berendsen, H. J. C. Gromacs User Manual version 4.5.4. Gromacs User Manual version 4.5.4, www.gromacs.org (2010)

(2) Bussi, G.; Donadio, D.; Parrinello, M. J. Chem. Phys. 2007, 126, 014101. 